\title{
A construção de capacidades e meios de vida na garantia da segurança alimentar do rural pernambucano
}

\author{
Amália Leonel Nascimento ${ }^{1}$, Fabiana Cristina Lima da Silva Pastich Gonçalves ${ }^{2}$, \\ Sandra Regina Maia ${ }^{3}$, Sérgio Schneider ${ }^{4}$, Pedro Israel Cabral de Lira ${ }^{5}$
}

\begin{abstract}
O trabalho teve como objetivo caracterizar a situação de (in)segurança alimentar do meio rural de Pernambuco e seus condicionantes, bem como, por meio da abordagem das capacidades e perspectiva dos meios de vida, buscar alternativas para a garantia da segurança alimentar e desenvolvimento rural do Estado. A insegurança alimentar na área rural de Pernambuco esteve relacionada à classe social, embora que não tão dependente da renda quanto no setor urbano. A localização geográfica e áreas de povoamento, bem como às características do chefe da familia e/ou entrevistado (gênero, condições de trabalho, escolaridade), presença de criança e/ou adolescente no domicílio, número de refeições realizadas e acesso a saneamento básico, tratamento de água, participação em programas sociais e cooperativas também demonstraram associação. As informações sobre insegurança alimentar no rural pernambucano auxiliam na compreensão das mais variadas formas de vulnerabilidade e exclusão sofridas por essas famílias, bem como suas estratégias para a construção de capacidades e ampliação da qualidade e meios de vida no campo.
\end{abstract}

Palavras-chave: Segurança Alimentar e Nutricional, desenvolvimento rural, abordagem das capacidades, meios de vida, atores sociais.

\section{Building capacities, livelihoods and food security of rural Pernambuco, Brazil}

The previously mentioned study aimed to characterize the situation of food (in)security in rural areas of Pernambuco and conditioning as well as through the capacities approach and perspective of livelihoods, to find alternatives to food security and rural development of State. Food insecurity in rural areas of Pernambuco was related not only to income level, measured by social class, but also the geographic location within the development regions of the State or populated areas, as well as characteristics of household head and/or interviewed such as gender, working conditions, education, self-reported color, presence of child/adolescent and number of meals and access to sanitation, water treatment and social programs. Information on food insecurity in rural Pernambuco assist in the understanding of various forms of vulnerability and exclusion these families as well as their strategies for capacity building and expansion of quality and livelihoods in rural areas.

Key-words: Food and Nutrition Security, rural development, capability approach, livelihoods, social actors.

\footnotetext{
${ }^{1}$ Doutoranda do Programa de Pós-Graduação em Desenvolvimento Rural, Universidade Federal do Rio Grande do Sul (UFRGS). E-mail: amalialeonel@gmail.com.

${ }^{2}$ Doutoranda do Programa de Pós-graduação em Saúde da Criança e do Adolescente, Universidade Federal de Pernambuco (UFPE).

${ }^{3}$ Mestre e Economista do Departamento de Nutrição, UFPE.

${ }^{4}$ Doutor e Professor do Departamento de Sociologia, UFRGS.
}

${ }^{5}$ Doutor e Professor do Departamento de Nutrição, UFPE. Correspondência: Departamento de Nutrição. Centro de Ciências da Saúde, UFPE. Av. Prof. Moraes Rego, 1235. CEP 50 670-601 - Recife, PE. Fone/Fax: (81) 21268470/2126 8476 


\section{INTRODUÇÃO}

Embora seja ainda um desafio, a promoção da Segurança Alimentar e Nutricional (SAN) e sua intersetorialidade, inclusive nos processos de produção de conhecimento, vem sendo discutida como um direito humano a ser atendido a partir de referenciais éticos e envolvendo questões estruturais, como o enfrentamento às desigualdades sociais e econômicas em vários setores: política econômica, emprego e renda, políticas de produção agroalimentar, comercialização, distribuição, acesso e consumo de alimentos, com perspectivas de descentralização e diferenciação regional para o desenvolvimento $[1,2]$.

Nesse contexto, o desenvolvimento rural surge como uma combinação dos aspectos sociais e econômicos no campo, tão marcado por essas desigualdades, e como forma de associar atividade e território do ponto de vista econômico e social, observando a capacidade de inserção nas economias locais e regionais, de como se dá a interação e integração das famílias, domicílios, indivíduos ou empresas nas redes econômicas e sociais locais [3].

A intersetorialidade da segurança alimentar e o caráter multifacetado do desenvolvimento rural permitem não só abordagens teóricas das mais diversas, mas postos em prática, são conceitos que exigem ações articuladas, que identifiquem determinantes-chave do problema em pauta, formulem intervenções estratégicas que transcendem os programas setoriais e aloquem os recursos em função dessas prioridades, para melhoria da qualidade de vida e do bem-estar das populações $[4,5]$.

No intuito de centrar essa construção nos atores, surge a perspectiva da Abordagem das Capacidades de Amartya Sen, que, por meio do exercício da liberdade, aborda o tema sob múltiplas dimensões, sugerindo que a pobreza deve ser entendida como a falta de capacitações para desenvolver funcionamentos [ $[$. A aplicação de suas bases teóricas ao Desenvolvimento Rural por Frank Ellis contribui para a compreensão das estratégias e alternativas que esses atores constroem para diversificar seus meios de vida e permitir a sustentabilidade no campo, resistindo e se adaptando conforme as características e intensidade das situações [7].

$\mathrm{Na}$ análise das situações de insegurança alimentar e da pobreza da população brasileira, a abordagem seniana vem recebendo maior atenção por parte dos pesquisadores do tema, buscando congregar, em seus estudos, aspectos qualitativos e quantitativos, envolvendo aspectos econômicos, sociais e culturais, que se caracterizam por insuficiência de renda, carência ou acesso limitado a serviços de necessidades básicas e por exclusão social e discriminação devida à origem étnica ou gênero [8,9].

Sob o aspecto empírico, este trabalho procurou caracterizar a situação de (in)segurança alimentar do meio rural de Pernambuco e seus condicionantes. Sob aspecto teórico, buscou o respaldo na abordagem das capacidades e perspectiva dos meios de vida com vistas à promoção da segurança alimentar e o desenvolvimento rural do Estado. Não cabe aqui discutir a relação causal entre as variáveis e dimensões analisadas, mas sim a relevância da intersecção desses temas e o papel dos atores na sua construção.

\section{SEGURANÇA ALIMENTAR NA AGENDA DO DESENVOLVIMENTO RURAL}

A SAN no Brasil passou a ter um caráter estratégico para o desenvolvimento social, sendo a principal proposta de política governamental nas últimas décadas, pautada nos princípios do direito humano à alimentação adequada, soberania alimentar, intersetorialidade, equidade e participação social [10,11]. Tida como o fiel de uma balança, na medida que seu conceito passa pelos eixos político, social, coparticipativo, a SAN ressurge como um direito de cidadania ativa, numa visão holística, com inclusão econômica e social nos meios de produção, promoção da soberania alimentar, melhoria da qualidade de vida, preservação da cultura e meio ambiente ${ }^{[1,2,12]}$.

Vários são os aspectos da SAN que contribuem para uma nova perspectiva analítica. Tais aspectos permitem identificar as dimensões social, política e cultural contidas nos modos de produzir, distribuir e consumir alimentos, ressaltando o lugar da alimentação na identidade cultural dos indivíduos, comunidades e grupos populacionais ou povos como um direito de estarem livres da fome e decidirem o que/como querem produzir e comer, tendo um impacto significativo no desenvolvimento dessas comunidades ${ }^{[13-15]}$.

O papel da alimentação na construção das relações sociais, no que podemos chamar de "humanidade" - na sua diversidade e ligação intrínseca à identidade cultural - e do desenvolvimento como forma de liberdade tem contribuído para legitimar a perspectiva democrática da SAN como direito e 
questão fundamental nas estratégias de desenvolvimento humano [6,16]. E no contexto sóciohistórico brasileiro, que ainda negligencia e até deprecia as populações rurais, é necessária a inserção do tema desenvolvimento rural no debate relativo a SAN. E não basta dizer que o desenvolvimento rural é necessário. Ainda é preciso mostrar que ele é possível [17-19].

Nesse contraste histórico, o campo, embora em redução da proporção de domicílios com moradores em situação de insegurança alimentar (de 43,6\% em 2004 para 35,1\% em 2009), segundo Pesquisa Nacional por Amostra de Domicílios, ainda apresenta piores condições que o meio urbano, que diminuiu de 33,3\% para 29,4\% no mesmo período [20,21]. Segundo Sachs [19], um desenvolvimento rural socialmente includente e em harmonia com o meio ambiente exige tanto soluções intensivas em conhecimentos e mão de obra quanto econômicas em capital e recursos naturais e se desdobra num imperativo social e ecológico necessário e possível em meio às estruturas fundiárias desiguais com desaceleração do êxodo rural e humanização do campo e equilíbrio entre cidade-campo.

\section{MEDINDO A SEGURANÇA ALIMENTAR NO CAMPO: HÁ UMA ESCALA ADEQUADA AO RURAL?}

Com um conceito tão amplo e em evolução, a SAN não dispõe de nenhum indicador, isoladamente, que dê conta de suas múltiplas dimensões. Quando se discute SAN, o que está implícito é que haja condições de vida e condições nutricionais adequadas [22]. Para a dimensão da percepção da (in)segurança alimentar e da fome, por exemplo, uma avaliação subjetiva, mas direta, leva em conta que a fome é um problema tão social quanto biológico [23].

Indicadores qualitativos ou de "autoavaliação" $\mathrm{da}$ insegurança alimentar captam aspectos que mostram-se estreitamente relacionados com outras medidas de insegurança alimentar e fome [24]. Um exemplo é a Escala Brasileira de Insegurança Alimentar (EBIA), adaptada do instrumento americano Household Food Security Survey Modle, a qual analisa em 15 perguntas desde a preocupação e angústia frente à possibilidade de não dispor regularmente de alimentos até a percepção de problemas na adequação da dieta que, no limite, levaria à fome [22,25].
Apesar de validada em áreas urbanas e rurais das macrorregiões brasileiras, a EBIA apresenta algumas desvantagens, como divergências no seu período de aplicação (mensal ou anual) e especialmente suscetível a vieses de "prestígio" ou "benefício" se os entrevistados imaginam que, dependendo das respostas que emitirem, poderão receber ajuda em alimentos ou benefícios sociais. Assim, é fundamental que os indivíduos entrevistados saibam que o objetivo do estudo não se encontra atrelado a nenhum programa ou ação em SAN com benefícios diretos ou indiretos [25,26].

O baixo custo de aplicação da escala tem a grande vantagem de permitir a descentralização dos esforços governamentais no monitoramento e combate a insegurança alimentar; e deve ser usado como uma possibilidade de melhor compreensão do fenômeno da fome e nova oportunidade de parceria das universidades com a gestão da política pública. Se usada para determinar a elegibilidade em programas assistenciais sociais e alimentares, a escala perde sua validade [25].

A EBIA considera o consumo alimentar infantil como sinalizador de gravidade da situação de insegurança alimentar domiciliar, mas não pondera o privilégio muitas vezes dado ao chefe de família, uma vez que a garantia de sua alimentação conservaria seu trabalho do campo. Segundo Woortmann [27], as necessidades de produção do roçado e reprodução familiar determinam a quantidade de comida pertinente a cada membro segundo sua capacidade de trabalho, o que, diante de situações de pobreza, como uma privação de alimentos essenciais, afetaria primeiro as crianças em detrimento aos adultos.

Em relação ao meio rural, ainda, a escala não analisa a possibilidade de produção agrícola para autoconsumo e seus modos coletivos de produção (extrativismo, faxinais, fundos de pasto, assentamentos, etc.) ou práticas não mercantis de acesso aos alimentos (reciprocidade, trocas, dádivas). Questões sobre posse de terra, acesso à água ou a insumos agrícolas, origem dos alimentos, contaminação microbiológica ou com metais pesados e pesticidas não são capturadas, assim como a situação da safra atual no momento da aplicação da escala [28,29].

Sensível ao desenvolvimento social, econômico e às diversidades culturais, a escala difere, portanto, entre grupos populacionais e deve ser 
utilizada inserida nos instrumentos de coleta de informações das pesquisas. As autoras da escala brasileira (EBIA) advertem que novas dimensões tornam mais complexos os intentos de mensurar a segurança alimentar e atestam a necessidade de outras investigações sobre indicadores de medida de segurança alimentar, de preferência com abordagem multi e interdisciplinar, o que constitui novos desafios para pesquisadores e gestores das políticas públicas ${ }^{[30]}$.

No meio rural, se as investigações forem associadas a fatores socioeconômicos e de acesso a bens e serviços, poderão nos levar a uma compreensão de um processo tão abrangente quanto o é, permitindo o planejamento e a implementação de políticas públicas mais efetivas para a promoção da SAN e o desenvolvimento rural, de extrema importância para amenizar ou resolver índices sociais ainda negativos [28,31].

\section{INSEGURANÇA ALIMENTAR NAS REGIÕES DE DESENVOLVIMENTO DE PERNAMBUCO}

Um estudo transversal desenvolvido pela Universidade Federal de Pernambuco, por meio do Departamento de Nutrição e do Instituto de Pesquisas Sociais e Aplicadas, em parceria com a Superintendência Estadual das Ações de Segurança Alimentar e Nutricional dividiu o Estado em 12 Regiões de Desenvolvimento (RDs) para analisar a situação de insegurança alimentar e apontar as condições familiares que lhes impõe maior vulnerabilidade social [32].

Para efeito da regionalização das ações no âmbito estadual, quatro municípios por RDs e quatro setores censitários por município foram sorteados, com distribuição proporcional à população urbana e rural, segundo o Censo Demográfico [33] e, aproximadamente, 15 domicílios por setor censitário, totalizando 2.904 famílias para o Estado de Pernambuco como um todo. A medida da (in)segurança alimentar teve como unidade de análise o domicílio e a escala utilizada foi a EBIA associada a variáveis socioeconômicas referentes ao domicílio, ao chefe da família e à pessoa entrevistada, a fim de analisar as condições de acesso a bens e serviços essenciais nas regiões de desenvolvimento do Estado.

O sujeito da pesquisa foi o adulto responsável pelo domicílio no momento da entrevista, chefe ou não da família. Os dados foram digitados em dupla entrada, utilizando o programa estatístico Epi Info, versão 6.04 e as análises estatísticas, realizadas com o auxilio do Statistical Package for Social Science (SPSS), versão 12.0.1. A associação entre as variáveis, efetuada pelo teste quiquadrado de Pearson, considerou como nível de significância estatística valores de $p<0,05$. A localização geográfica do domicílio, setor urbano, foi utilizada como uma variável de controle para a associação entre a (in)segurança alimentar e o domicílio no setor rural.

Num total de 48 municípios pernambucanos pesquisados, distribuiu-se a amostra em 63,1\% na área urbana e 36,9\% na área rural. O índice de insegurança alimentar do Estado foi de 61,8\%, com proporções de $27,5 \%$ e $9,6 \%$ para as formas moderada e grave. Com relação às áreas geográficas, no meio rural foi observada maior proporção de insegurança alimentar, com 32,4\% e $11,6 \%$ das formas moderada e grave, estatisticamente significante ( $p$-valor $<0,0001)$, enquanto no setor urbano os valores encontrados foram de $24,6 \%$ e 8,5\%, respectivamente (Figura 1).

Com foco na área rural e considerando o critério da Associação Brasileira de Empresas de Pesquisa (ABEP) [34] de categorias de classe social, observou-se situação bastante diferenciada entre as formas extremas de insegurança alimentar (ausência e situação grave), inversamente proporcional à classe. Praticamente livres de insegurança alimentar moderada/grave, as classes sociais A e B apresentaram mais de dois terços de suas famílias rurais em segurança alimentar, enquanto que nas classes D e E, esse percentual não representa um quinto da população (Figura 2).

A fim de controlar o possível efeito da região de residência na probabilidade de insegurança alimentar segundo classe socioeconômica, foi realizada análise estratificada, uma vez que estudos verificaram que a residência rural e a atividade agrícola contribuem para reduzir a insegurança alimentar ${ }^{[8]}$. Considerando a classe A/B como referência, houve um aumento significativo do risco de insegurança alimentar nas demais classes no meio rural. Mas em comparação com o setor urbano, o risco para as classes desfavorecidas mostrou-se bem maior que no meio rural, principalmente nos estratos mais baixos (classe E e D), o que significa que outros fatores interfeririam mais fortemente que a renda na zona rural (Tabela 1). 
Figura 1. Distribuição da insegurança alimentar (\%) segundo localização geográfica do domić́lio, Pernambuco, 2011

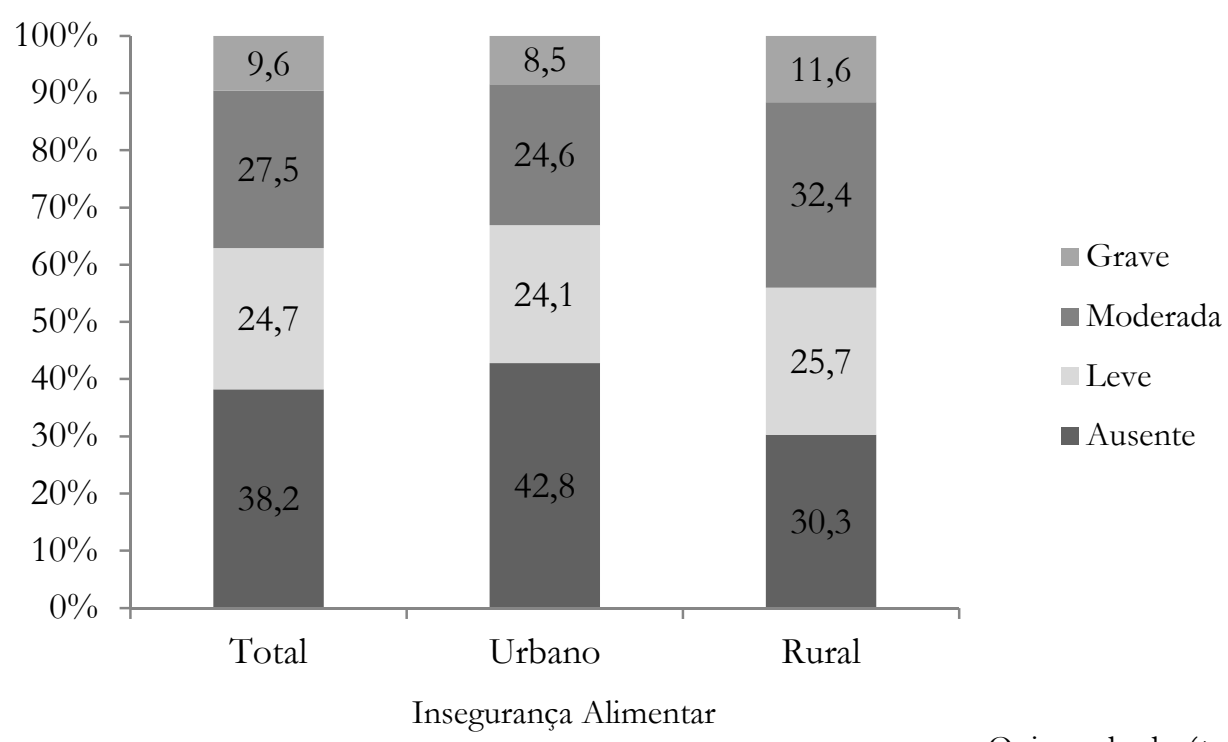

Qui-quadrado ( $p$-valor $<0,0001)$

Fonte: adaptado de Pernambuco, $2011^{[32]}$.

Figura 2. Distribuição da insegurança alimentar (\%) segundo a situação econômica familiar (Critério ABEP) na área rural, Pernambuco, 2011

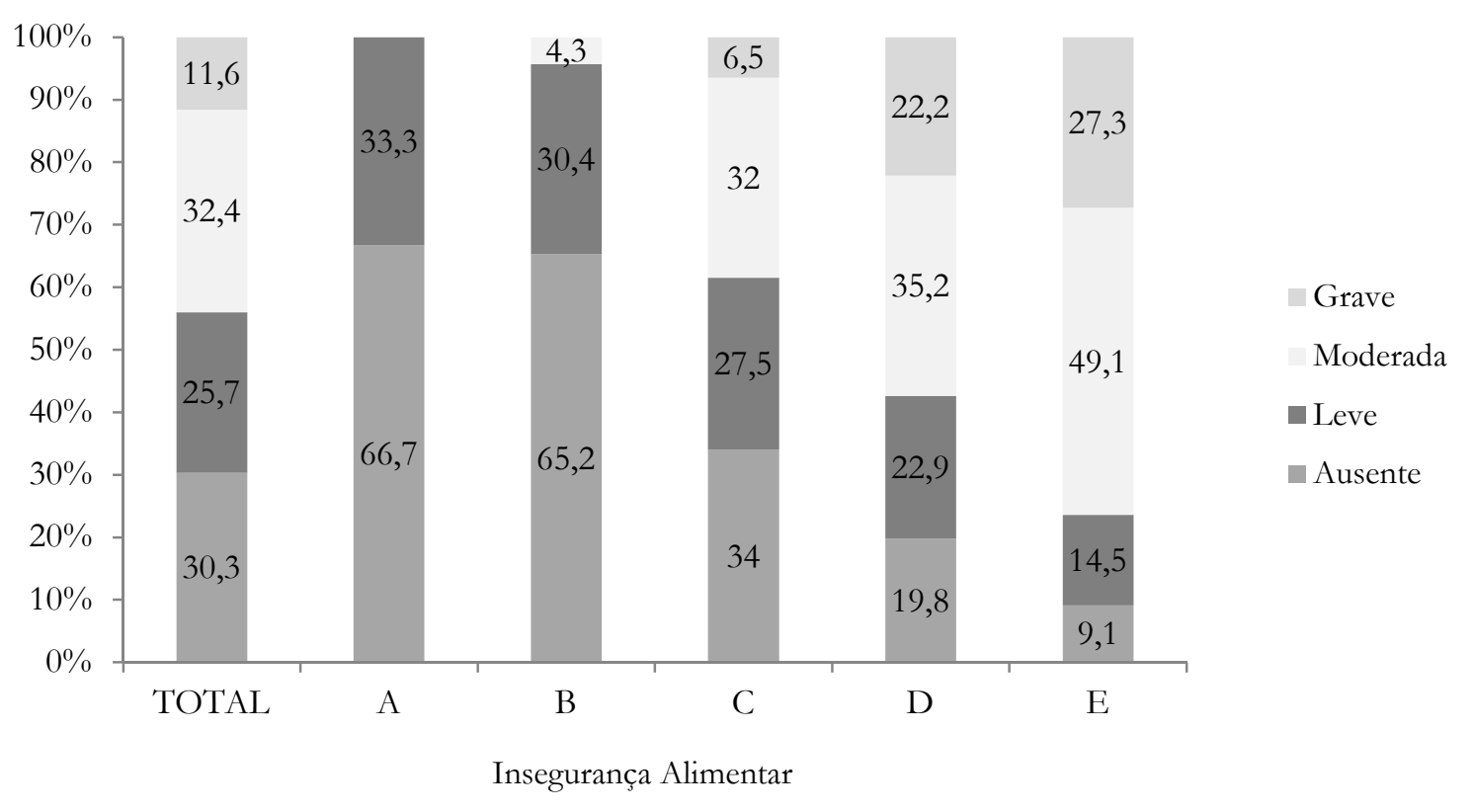

Qui-quadrado ( $p$-valor $<0,0001)$

Fonte: adaptado de Pernambuco, $2011^{[32]}$. 
Tabela 1. Associação entre insegurança alimentar e classe socioeconômica segundo localização geográfica do domicílio em Pernambuco, com estimação da razão de chances e intervalo de confiança de 95\%, 2011

\begin{tabular}{|c|c|c|c|c|}
\hline \multirow{2}{*}{$\begin{array}{c}\text { Insegurança } \\
\text { Alimentar }\end{array}$} & \multicolumn{4}{|c|}{ Classe Socioeconômica } \\
\hline & Classes A/B & Classe C & Classe D & Classe E \\
\hline \multicolumn{5}{|l|}{ Rural } \\
\hline Não & $32(9,8 \%)$ & $230(70,8 \%)$ & $58(17,8 \%)$ & $5(1,5 \%)$ \\
\hline $\operatorname{Sim}$ & $17(2,3 \%)$ & $446(59,6 \%)$ & $235(31,4 \%)$ & $50(6,7 \%)$ \\
\hline OR [IC 95\%]* & 1,0 & $3,65[1,90-7,06]$ & $7,63[3,76-15,59]$ & $18,82[5,68-66,71]$ \\
\hline \multicolumn{5}{|l|}{ Urbano } \\
\hline Não & $184(23,5 \%)$ & $559(71,3 \%)$ & $40(5,1 \%)$ & $1(0,1 \%)$ \\
\hline $\operatorname{Sim}$ & $72(6,9 \%)$ & $815(77,8 \%)$ & $144(13,8 \%)$ & $16(1,5 \%)$ \\
\hline OR [IC 95\%]* & 1,0 & $3,73[2,74-5,06]$ & $9,2[5,74-14,78]$ & $40,9[5,47-856,3]$ \\
\hline
\end{tabular}

Os dados da área rural das RDs observados na Tabela 2 foram ordenados pelo nível de insegurança alimentar grave. Assim sendo, as RDs rurais do Sertão do Moxotó (21,3\%), Agreste Central (18,7\%), Sertão do São Francisco $(14,3 \%)$ e Região Metropolitana do Recife $(14,1 \%)$ apresentaram os piores índices, como as áreas de assentamento e povoamento indígena e quilombola, ainda que agrupadas.

Em relação às características da família, a insegurança alimentar no meio rural foi mais grave e estatisticamente significante quando o chefe da familia era do gênero feminino, não trabalhava ou tinha trabalho informal e não concluiu ao menos o primeiro grau ou ginasial (Tabela 3).

Ainda, a insegurança alimentar apresentou-se maior quanto piores as condições de saneamento básico, abastecimento de água, qualidade e tratamento da água de beber na área rural de Pernambuco, destacando-se vala ou esgoto a céu aberto e água de beber sem tratamento eficaz, conforme Tabela 4.
A insegurança alimentar agravou-se em famílias rurais com crianças menores de 5 anos ou com moradores menores de 18 anos de idade, sendo as formas moderada e grave similares nos dois casos. Ao analisar a insegurança alimentar em relação ao número de refeições diárias dos domićlios pesquisados, observou-se que a forma grave era cinco vezes maior nas famílias que relataram apenas 1 ou 2 refeições principais por dia, quando comparadas àquelas com três e mais. Ou seja, além do medo detectado pela EBIA, essas familias de fato não realizam as três refeições principais diárias, o mínimo recomendado pelo Ministério da Saúde (Tabela 5).

A distribuição da insegurança alimentar em relação ao acesso aos programas sociais, de benefício de prestação continuada (aposentados, idosos ou deficientes) e participação em cooperativas encontramse na Tabela 6. A insegurança alimentar para as familias com acesso a um ou mais programas sociais foi maior quando comparada às famílias que não participam dessas ações. Para o Programa Bolsa 
Família, com maior percentual de usuários, a insegurança moderada e grave também foram maiores. Esse resultado poderia levantar polêmica sobre uma suposta baixa eficiência do programa. Entende-se, entretanto, como mais plausível, que a população de seus usuários pode concentrar condições outras de vulnerabilidade que reduzam o impacto da transferência de renda [30].

Para os demais programas na área rural, os resultados foram semelhantes, mas o tamanho amostral das famílias participantes foi considerado ainda pequeno e as categorias de insegurança alimentar devem ser analisadas com cautela. A participação em cooperativas também apresentou diferença estatística, estando mais presente nas famílias em piores condições de insegurança alimentar grave, provavelmente como alternativa para inserção coletiva e melhoria nas condições de produção e comercialização, diminuindo os riscos e possibilitando maior agregação de valor para produtores rurais em condições desfavoráveis nas relações de mercados concentrados (Tabela 6) [35].

Tabela 2. Distribuição da Insegurança Alimentar (\%) segundo Regiões de Desenvolvimento (RDs) em áreas rurais e de povoamento em Pernambuco, 2011

\begin{tabular}{|c|c|c|c|c|}
\hline \multirow{3}{*}{$\begin{array}{l}\text { Regiões de Desenvolvimento } \\
\qquad(p \text {-valor }<0,0001)^{*}\end{array}$} & \multicolumn{4}{|c|}{ Insegurança Alimentar (\%) } \\
\hline & Ausente & Leve & Moderada & Grave \\
\hline & $(n=325)$ & $(n=276)$ & $(n=348)$ & $(n=124)$ \\
\hline 10 Sertão do Moxotó & 27,0 & 16,9 & 34,8 & 21,3 \\
\hline 2o Agreste Central & 29,3 & 20,0 & 32,0 & 18,7 \\
\hline 3o Sertão do São Francisco & 21,9 & 21,9 & 41,9 & 14,3 \\
\hline 4o Área de Assentamento \Quilombo \Indígena & 21,9 & 29,4 & 34,3 & 14,3 \\
\hline 5o Região Metropolitana & 28,1 & 18,8 & 39,1 & 14,1 \\
\hline 6o Sertão do Araripe & 34,1 & 28,5 & 26,0 & 11,4 \\
\hline 70 Agreste Meridional & 42,2 & 20,0 & 26,7 & 11,1 \\
\hline 80 Sertão Central & 27,8 & 24,4 & 37,8 & 10,0 \\
\hline 9o Mata Norte & 35,9 & 18,8 & 35,9 & 9,4 \\
\hline $10^{\circ}$ Mata Sul & 30,7 & 26,7 & 33,3 & 9,3 \\
\hline 11으 Sertão de Itaparica & 20,0 & 37,8 & 33,3 & 8,9 \\
\hline 12으 Agreste Setentrional & 43,7 & 26,1 & 23,5 & 6,7 \\
\hline 13ㅇ Sertão do Pajeú & 19,1 & 43,8 & 31,5 & 5,6 \\
\hline
\end{tabular}

*Qui-quadrado. Fonte: adaptado de Pernambuco, $2011^{[32]}$. 
Tabela 3. Distribuição da Insegurança Alimentar (\%), segundo gênero, escolaridade e situação ocupacional do chefe da familia na área rural de Pernambuco, 2011

\begin{tabular}{|c|c|c|c|c|c|}
\hline \multirow{3}{*}{$\begin{array}{l}\text { Características } \\
\text { do chefe da família }\end{array}$} & \multicolumn{5}{|c|}{ Insegurança Alimentar (\%) } \\
\hline & \multirow{2}{*}{$\begin{array}{l}\text { Ausente } \\
(n=325)\end{array}$} & \multirow{2}{*}{$\begin{array}{c}\text { Leve } \\
(n=276)\end{array}$} & \multirow{2}{*}{$\begin{array}{c}\text { Moderada } \\
(n=348)\end{array}$} & \multirow{2}{*}{$\begin{array}{c}\text { Grave } \\
(n=124)\end{array}$} & \multirow{2}{*}{$p$-valor } \\
\hline & & & & & \\
\hline Gênero & & & & & 0,015 \\
\hline Masculino & 32,6 & 25,7 & 31,5 & 10,1 & \\
\hline Feminino & 24,3 & 25,7 & 34,9 & 15,1 & \\
\hline Condição de Trabalho & & & & & $<0,0001$ \\
\hline Trabalho Formal & 41,9 & 27,9 & 25,0 & 5,1 & \\
\hline Trabalho Informal & 23,8 & 26,0 & 36,5 & 13,7 & \\
\hline Aposentado & 42,0 & 25,3 & 26,3 & 6,4 & \\
\hline Desempregado & 19,1 & 23,5 & 36,8 & 20,6 & \\
\hline Escolaridade & & & & & 0,003 \\
\hline Analfabeto/Primário Incompleto & 30,2 & 21,9 & 34,9 & 13,1 & \\
\hline Primário Completo/Ginasial Incompleto & 26,4 & 30,3 & 31,2 & 12,1 & \\
\hline Ginasial Completo/Colegial Incompleto & 35,0 & 25,0 & 35,0 & 5,0 & \\
\hline Colegial Completo/Superior Incompleto & 40,0 & 35,0 & 20,0 & 5,0 & \\
\hline Superior Completo & 53,8 & 30,8 & 15,4 & 0,0 & \\
\hline
\end{tabular}

* Qui-quadrado. Fonte: adaptado de Pernambuco, 2011 ${ }^{[32]}$.

\section{A CONSTRUÇÃO DE CAPACIDADES E MEIOS DE VIDA (LIVELIHOODS) EM PERNAMBUCO}

É um erro julgar a natureza e a gravidade dos problemas de insegurança alimentar (fome crônica, coletiva, subnutrição) apenas da perspectiva da produção de alimentos, inclusive quando se trata de desenvolvimento rural. É importante ver a produção de alimentos como resultado da atuação humana e compreender os incentivos que influenciam as decisões e ações dos indivíduos ${ }^{6]}$.
Ainda, uma dificuldade operacional importante é o uso de indicadores de renda, referidos ou não às linhas de pobreza e indigência, para seleção dos indivíduos elegíveis à participação em programas sociais, pois fornecem diferentes estimativas de população alvo aos programas governamentais [30]. $\mathrm{O}$ economista Amartya Sen ${ }^{[6]}$ defende que, para buscar um melhor entendimento da natureza e das causas da pobreza e privação das capacidades, é necessário basear-se na privação de capacidades humanas básicas para os fins que as pessoas têm razão para buscar e, correspondentemente, para as liberdades de poder alcançar esses fins - tanto as liberdades políticas como os direitos civis. 
Tabela 4. Distribuição da Insegurança Alimentar (\%) segundo a situação de saneamento e abastecimento d'água na área rural de Pernambuco, 2011

\begin{tabular}{|c|c|c|c|c|c|}
\hline \multirow{3}{*}{$\begin{array}{c}\text { Características do } \\
\text { domicílio rural }\end{array}$} & \multicolumn{5}{|c|}{ Insegurança Alimentar (\%) } \\
\hline & Ausente & Leve & Moderada & Grave & \\
\hline & $(n=325)$ & $(n=276)$ & $(n=348)$ & $(n=124)$ & $p$-valor* \\
\hline Esgotamento sanitário & & & & & $<0,0001$ \\
\hline Rede Pública & 34,9 & 25,6 & 32,6 & 7,0 & \\
\hline Fossa séptica ou rudimentar & 34,7 & 26,8 & 30,8 & 7,7 & \\
\hline Vala/Céu aberto & 20,1 & 23,5 & 35,8 & 20,7 & \\
\hline Abastecimento de água & & & & & 0,535 \\
\hline Rede pública & 29,5 & 27,0 & 33,1 & 10,3 & \\
\hline Poço/Cacimba/Barreiro & 28,5 & 25,9 & 32,6 & 13,0 & \\
\hline Cisterna/Chuva & 35,5 & 23,2 & 30,9 & 10,5 & \\
\hline Outro & 29,5 & 27,0 & 33,1 & 10,3 & \\
\hline Água de beber & & & & & 0,001 \\
\hline Tratada & 32,8 & 28,1 & 29,9 & 9,2 & \\
\hline Sem tratamento & 26,8 & 22,1 & 36,3 & 14,9 & \\
\hline Tratamento água beber & & & & & $<0,0001$ \\
\hline Mineral & 57,3 & 25,6 & 13,4 & 3,7 & \\
\hline Filtrada/Fervida & 32,5 & 30,5 & 33,1 & 4,0 & \\
\hline Clorada & 28,0 & 27,5 & 32,5 & 12,0 & \\
\hline Outro (misto, coada) & 23,8 & 38,1 & 19,0 & 19,0 & \\
\hline Sem tratamento & 26,8 & 22,1 & 36,3 & 14,9 & \\
\hline
\end{tabular}

*Qui-quadrado. Fonte: adaptado de Pernambuco, $2011^{[32]}$. 
Tabela 5. Distribuição da Insegurança Alimentar (\%) segundo faixa etária dos moradores e número de refeições diárias na área rural de Pernambuco, 2011

\begin{tabular}{|c|c|c|c|c|c|}
\hline \multirow{3}{*}{ Características das famílias rurais } & \multicolumn{5}{|c|}{ Insegurança Alimentar (\%) } \\
\hline & \multirow{2}{*}{$\begin{array}{c}\text { Ausente } \\
(n=325)\end{array}$} & \multirow{2}{*}{$\begin{array}{c}\text { Leve } \\
(n=276)\end{array}$} & \multirow{2}{*}{\begin{tabular}{|c|} 
Moderada \\
$(n=348)$
\end{tabular}} & \multirow{2}{*}{$\begin{array}{c}\text { Grave } \\
(n=124)\end{array}$} & \multirow{2}{*}{$p$-valor* } \\
\hline & & & & & \\
\hline Com crianças $<5$ anos & & & & & $<0,0001$ \\
\hline Não & 34,5 & 23,9 & 31,4 & 10,3 & \\
\hline Sim & 20,8 & 30,0 & 34,9 & 14,4 & \\
\hline Com moradores $<18$ anos & & & & & $<0,0001$ \\
\hline Não & 45,1 & 23,0 & 26,6 & 5,3 & \\
\hline $\operatorname{Sim}$ & 22,2 & 27,2 & 35,6 & 15,0 & \\
\hline № de refeições principais & & & & & $<0,0001$ \\
\hline 1 ou 2 & 9,4 & 18,8 & 37,5 & 34,4 & \\
\hline 3 ou mais & 31,6 & 26,2 & 32,1 & 10,1 & \\
\hline № de lanches & & & & & $<0,0001$ \\
\hline 0 & 16,5 & 16,5 & 45,7 & 21,4 & \\
\hline 1 & 31,8 & 32,5 & 28,2 & 7,5 & \\
\hline 2 ou mais & 47,1 & 27,4 & 20,9 & 4,6 & \\
\hline
\end{tabular}

*Qui-quadrado. Fonte: adaptado de Pernambuco, 2011 ${ }^{[32]}$.

A luta defendida por Josué de Castro pela adoção de um modelo de desenvolvimento humano sustentável e uma sociedade sem miséria e sem fome é hoje, reacendida pelo compromisso de Sen por um desenvolvimento, na perspectiva da liberdade, pela redução da desigualdade e da privação de capacidades, acentuada pela idade da pessoa, pelos papéis sexuais e sociais, pela localização - a exemplo do rural - e outras condições epidemiológicas [6,36].

Assim, tanto questões descritivas como questões de políticas podem ser tratadas por meio dessa perspectiva mais ampla sobre desigualdade e pobreza segundo a privação de capacidades. A participação pública nesses debates seria uma parte crucial do exercício da democracia e escolha social responsável, assim como o uso de prerrogativas democráticas ${ }^{[}$.

Segundo Pereira et al. [37], a maior inovação conceitual de Amartya Sen, seria a utilização da palavra "capacidades", a qual teria a finalidade de separar a habilidade para satisfazer certos funcionamentos crucialmente importantes até certos níveis adequadamente mínimos. Ellis [7], ao aplicar suas bases teóricas ao desenvolvimento rural através do conceito de livelihoods (meios de vida), criados por Chambers \&
Conway [38], também credita a Sen [39] a definição do termo "capacidades", referindo-se à habilidade que os indivíduos têm para desenvolver seu potencial como seres humanos.

Complementando o conceito de Chambers \& Conway ${ }^{[3]}$, Ellis ${ }^{[7]}$ atribui maior força às questões de acesso, particularmente considerando importante $\mathrm{O}$ impacto das relações sociais e das instituições que mediam a capacidade individual ou familiar para alcançar suas necessidades de consumo. Scoones [40] reconhece ainda importantes contribuições de economistas marxistas, particularmente nos campos da agricultura e geografia econômica, de onde uma perspectiva livelihoods transdisciplinar influenciou profundamente o pensamento e prática do desenvolvimento rural.

Tem-se, assim, a conjunção das capacidades, dos ativos e sustentabilidade, em um só conceito na referência dos meios de vida [34]. É por meio dessa abordagem e dos dados anteriormente apresentados da situação de insegurança alimentar na zona rural de Pernambuco que o referido trabalho vem buscar alternativas para a construção de capacidades e meios de vida (livelihoods) na garantia da segurança alimentar no rural pernambucano, conhecendo a complexidade 
da realidade de vida dos cidadãos e diminuindo as privações de liberdade a que as pessoas são submetidas [41].

\section{CONSIDERAÇÕES FINAIS}

A insegurança alimentar na área rural de Pernambuco esteve relacionada à classe social, embora que não tão dependente da renda quanto o setor urbano. A localização geográfica dentro das regiões de desenvolvimento do Estado e áreas de povoamento, bem como às características do chefe da família e/ou entrevistado (gênero, condições de trabalho, escolaridade), presença de criança e/ou adolescente no domicílio, número de refeições realizadas e acesso a saneamento básico, tratamento de água, participação em programas sociais e cooperativas também demonstraram associação com a insegurança alimentar no meio rural.

No que diz respeito aos princípios que pautam a realização de uma alimentação de qualidade e direito no rural pernambucano, questões como desigualdade entre as classes sociais e regiões de desenvolvimento, baixa escolaridade, desemprego e condições precárias de moradia foram apresentadas neste estudo. As informações sobre insegurança alimentar no rural pernambucano auxiliam na compreensão das mais variadas formas de vulnerabilidade e exclusão sofridas por essas famílias, bem como suas estratégias para a construção de capacidades e ampliação da qualidade e meios de vida no campo.

Tabela 6. Distribuição da Insegurança Alimentar (\%) segundo acesso da família a programas sociais na área rural de Pernambuco, 2011

\begin{tabular}{l|c|c|c|c|c}
\hline \multirow{2}{*}{\multicolumn{1}{c|}{ Programas sociais }} & \multicolumn{5}{c}{ Insegurança Alimentar (\%) } \\
\cline { 2 - 5 } & Ausente & Leve & Moderada & Grave & \multirow{2}{*}{$\boldsymbol{p}$-valor * } \\
\cline { 2 - 5 } & $\mathbf{( n = 3 2 5 )}$ & $(\boldsymbol{n}=\mathbf{2 7 6})$ & $\mathbf{( n = 3 4 8 )}$ & $\mathbf{( n = 1 2 4 )}$ & \\
\hline Acesso a pelo menos um programa & 24,4 & 25,8 & 36,5 & 13,3 & $<0,0001$ \\
Não & 43,4 & 25,4 & 23,4 & 7,8 & \\
Bolsa Família & 18,2 & 27,5 & 38,9 & 15,4 & \multirow{2}{*}{$<0,0001$} \\
Não & 44,4 & 23,6 & 24,8 & 7,1 & \\
Cesta de Alimentos & 16,7 & 16,7 & 16,7 & 50,0 & \multirow{2}{*}{0,033} \\
Não & 30,4 & 25,8 & 32,5 & 11,3 & \\
Peti & 20,0 & 13,3 & 60,0 & 6,7 & 0,150 \\
Não & 30,4 & 25,9 & 32,0 & 11,6 & \\
Projeto Cisternas & 36,8 & 21,3 & 27,1 & 14,8 & 0,066 \\
Não & 29,2 & 26,5 & 33,3 & 11,0 & \\
Pronaf & 4,5 & 40,9 & 27,3 & 27,3 & 0,008 \\
Não & 30,8 & 25,4 & 32,5 & 11,2 & \\
Programa Aquisição de Alimentos & 16,4 & 31,3 & 29,9 & 22,4 & 0,005 \\
Não & 31,2 & 25,4 & 32,5 & 10,8 & \\
Benefício de Prestação Continuada & 51,1 & 18,2 & 27,0 & 3,6 & $<0,0001$ \\
Não & 27,2 & 26,8 & 33,2 & 12,7 & \\
Participação em Cooperativas & 30,8 & 22,3 & 31,3 & 15,6 & 0,004 \\
Não & 38,8 & 24,9 & 27,2 & 9,1 & \\
\hline
\end{tabular}

* Qui-quadrado. Fonte: adaptado de Pernambuco, 2011 ${ }^{[32]}$. 
Cabe ressalvar que este trabalho não teve a pretensão de esgotar as possibilidades de análise e interpretação da situação de insegurança alimentar das familias rurais pernambucanas. Foi papel adicional dele, todavia, aquecer antigos debates e fomentar novas demandas para a temática em questão. Ainda são necessários estudos que analisem a evolução histórica dessas regiões rurais do Estado, bem como das condições de vida, hábitos alimentares, produção de alimentos e outros aspectos relacionados à insegurança alimentar no campo.

Faz-se necessário e urgente um olhar diferenciado e coletivo à complexidade do rural pernambucano e suas pluralidades para a elaboração de políticas em uma estrutura democrática, a partir da discussão pública e participação social no meio rural. E nessa ótica, a questão da segurança alimentar em Pernambuco passa a representar um elemento central das estratégias de desenvolvimento rural do Estado, não apenas com o foco no fornecimento de alimentos, mas na luta pela inclusão econômica e social nos meios de produção, promoção da soberania alimentar, melhoria da qualidade de vida, preservação da cultura e resgate da cidadania no campo.

\section{REFERÊNCIAS}

[1] Castro LMC. Pesquisar sobre segurança alimentar e nutricional no Brasil: a que viemos?. Ciênc Saúde Colet. 2010;15(1):26-8.

[2] Prado SD, Gugelmin SA, Mattos RA, Silva JK, Olivares PSG. A pesquisa sobre segurança alimentar e nutricional no Brasil de 2000 a 2005: tendências e desafios. Ciênc Saúde Colet. 2010:15(1):7-18.

[3] Kageyama A. Desenvolvimento rural: conceito e medida. CC\&T. 2004;21(3):379-408.

[4] Schneider S. A abordagem territorial do desenvolvimento rural e suas articulações externas. Sociologias. 2004;s/v(11):88-125.

[5] Burlandy L. A construção da política de segurança alimentar e nutricional no Brasil: estratégias e desafios para a promoção da intersetorialidade no âmbito federal de governo. Ciênc Saúde Colet. 2009;14(3):851-60.

[6] Sen AK. Desenvolvimento como liberdade. São Paulo: Companhia das Letras; 2000.

[7] Ellis F. Rural livelihoods and diversity in developing countries. Oxford: Oxford University Press; 2000.
[8] Hoffmann R, Kageyama AA. Pobreza, insegurança alimentar e pluriatividade no Brasil. Teoria e Evidência Econômica. 2007;14(29):9-35.

[9] Fritz K, Waquil PD, Mattos E. A insegurança alimentar no Rio Grande do Sul: uma análise comparativa entre o rural e o urbano. In: 4o ENCONTRO DE ECONOMIA GAÚCHA, 2008, Porto Alegre. Anais... Porto Alegre: EDIPUCRS, 2008. p. 1-20.

[10] Belik W. Perspectivas para segurança alimentar e nutricional no Brasil. Saude Soc. 2003;12(1):12-20.

[11] Brasil. Lei no 11.346, de 15 de setembro de 2006. Cria o Sistema Nacional de Segurança Alimentar e Nutricional (SISAN) com vistas em assegurar o direito humano à alimentação e dá outras providências. Diário Oficial da União, Brasília, 18 set. 2006. Seção 1, p. 1.

[12] Nascimento AL, Andrade SLLS. Segurança alimentar e nutricional: pressupostos para uma nova cidadania? Cien Cult. 2010;62(4):34-38.

[13] Brasil. Conselho Nacional de Segurança Alimentar e Nutricional. II Conferência Nacional de Segurança Alimentar e Nutricional. A construção do conceito de segurança alimentar e nutricional. Brasília: CONSEA; 2004.

[14] Freitas MCS, Pena PGL. Segurança alimentar e nutricional: a produção do conhecimento com ênfase nos aspectos da cultura. Rev Nutr. 2007;20(1):69-81.

[15] Maluf RS. Segurança Alimentar e Nutricional como valorização da cultura alimentar. In: Miranda DS, Cornelli G. (Org.). Cultura e Alimentação: saberes alimentares e sabores culturais. São Paulo: SESC; 2007. p. 143-50.

[16] Valente FLS. Do combate à fome à segurança alimentar e nutricional: o direito à alimentação adequada. In: Valente FLS. Direito Humano à Alimentação: desafios e conquistas. São Paulo: Cortez Editora; 2002. p. 37-70.

[17] Abramovay R. O futuro das regiões rurais. Porto Alegre: Editora da UFRGS; 2003.

[18] Menasche R, Marques FC, Zanetti C. Autoconsumo e segurança alimentar: a agricultura familiar a partir dos saberes e práticas da alimentação. Rev Nutr. 2008;21(Supl.):145-58.

[19] Sachs I. Barricadas de ontem, campos de futuro. Estud Av. 2010;24(68):25-38.

[20] Instituto Brasileiro de Geografia e Estatística. Pesquisa Nacional por Amostra de Domicílios: segurança alimentar 2004. Rio de Janeiro: IBGE; 2009. 
[21] Instituto Brasileiro de Geografia e Estatística. Pesquisa Nacional por Amostra de Domicilios. Segurança Alimentar: 2004/2009. Rio de Janeiro: IBGE; 2010.

[22] Segall-Corrêa AM. Insegurança alimentar medida a partir da percepção das pessoas. Estud Av. 2007;21(60):143-154.

[23] Vannier-Santos MC, Pessanha L, Barbosa MTS. Segurança Alimentar e Nutricional e Indicadores SócioEconômicos. In: Seminário População, Pobreza e Desigualdade, 2007, Belo Horizonte. Anais... Belo Horizonte: ABEP, 2007. p. 1-21.

[24] Food Insecurity and Vulnerability Information and Mapping Systems (FIVIMS). Measurement and Assessment of Food Deprivation and Undernutrition. Proceedings of the International Scientific Symposium. Roma: FAO; 2002.

[25] Perez-Escamilla R, Segall-Corrêa AM. Food insecurity measurement and indicators. Rev Nutr. 2008;21(Supl.):15-26.

[26] Pereira DA, Vieira VL, Fiore EG, Cervato-Mancuso AM. Insegurança alimentar em região de alta vulnerabilidade social da cidade de São Paulo. Seg Alim Nutr. 2006;13(2):3442.

[27] Woortmann K. O sentido simbólico das práticas alimentares. In: Araújo WMC, Tenser CMR. (Org.). Gastronomia: cortes e recortes. Brasilia: SENAC; 2006. p. 23-55.

[28] Nascimento AL, Lira PIC, Maia SR. Posse da terra e produção de alimentos em domicílios com insegurança alimentar na Zona da Mata e Semiárido brasileiros. In: Gehlen VRF, Lainé PCV. (Org.). Costurando com fios invisíveis: a fragmentação do território rural. Recife: Editora Universitária da UFPE; 2012. p. 361-75.

[29] Sampaio MFA, Kepple AW, Segall-Corrêa AM, Oliveira JTA, Panigassi G, Maranha LK, et al. (In) Segurança Alimentar: experiência de grupos focais com populações rurais do Estado de São Paulo. Seg Alim Nutr. 2006;13(1):6477.

[30] Segall-Corrêa AM, Marin-Leon L. A Segurança Alimentar no Brasil: proposição e usos da Escala Brasileira de Medida da Insegurança Alimentar (EBIA) de 2003 a 2009. Seg Alim Nutr. 2009;16(2):1-19.
[31] Kepple AW, Segall-Corrêa AM. Conceituando e medindo segurança alimentar e nutricional. Ciênc Saúde Colet. 2011;16(1):187-99.

[32] Pernambuco (Estado). Secretaria de Desenvolvimento Social e Direitos Humanos. Superintendência das Ações de Segurança Alimentar e Nutricional. Avaliação da (In)Segurança Alimentar nas Regiões de Desenvolvimento do Estado de Pernambuco. Recife; 2011 [acesso em 2012 maio 03]. Disponível em: http://www2.sedsdh.pe.gov.br/web/sedsdh/secretaria/exe cutivas/sedas/suasan

[33] Instituto Brasileiro de Geografia e Estatística. Censo Demográfico. 2000 [acesso em 2010 jan 15]. Disponível em: http://www.ibge.gov.br

[34] Associação Brasileira de Empresas de Pesquisa. Critério de Classificação Econômica Brasil. 2010 [acesso em 2011 abr 01]. Disponível em: http://www.abep.org

[35] Viegas IFP. Comércio justo e segurança alimentar. Seg Alim Nutr. 2010:17(1):133-143.

[36] Castro J. Geografia da Fome. Rio de Janeiro: Civilização Brasileira; 1984.

[37] Pereira MA, Souza M, Schneider S. Meios de vida e livelihoods: aproximações e diferenças conceituais. Revista IDeAS. 2010;4(1):203-24.

[38] Chambers R, Conway G. Sustainable rural livelihoods: practical concepts for the 21 st century. IDS discussion paper, 296. Brighton: IDS; 1992.

[39] Sen AK. Capacidad y bienestar. In: Nussbaum M, Sen AK. (Org.). La calidad de vida. Ciudad de Mexico: Fondo de Cultura Económica, 1996. p. 54-83.

[40] Scoones I. Livelihoods perspectives and rural development. J Peasant Stud. 2009;36(1):171-96.

[41] Kuhn D, Waquil PD, Costa AM, Mattos E, Fritz K, Gianluppi L. Pobreza no Rio Grande do Sul: a heterogeneidade revelada pela abordagem das capacitações nos municípios gaúchos. Teoria e Evidência Econômica. 2006;14(26):113-34. 